Chirurg 2014 · 85:917

DOI 10.1007/s00104-014-2889-5

Online publiziert: 20. September 2014

(c) Springer-Verlag Berlin Heidelberg 2014

J. Reibetanz • C.-T. Germer

Klinik für Allgemein-, Viszeral-, Gefäß- und Kinderchirurgie, Universitätsklinikum Würzburg

\title{
Restaging beim Rektumkarzinom nach neoadjuvanter Radio-Chemo-Therapie mittels MRT und Endosonographie
}

zirkumferenzielle Resektionsrand (CRM+ vs. CRM-), wobei das histopathologische Tumorstadium des Resektates als Referenz galt.

Zhao RS, Wang H, Zhou ZY et al (2014) Restaging of locally advanced rectal cancer with magnetic resonance imaging and endoluminal ultrasound after preoperative chemoradiotherapy: a systemic review and meta-analysis. Dis Colon Rectum 57:388-395

\section{Hintergrund}

Ein exaktes lokales Restaging nach neoadjuvanter Radio-Chemo-Therapie (RCT) beim lokal fortgeschrittenen Rektumkarzinom ist u. a. wichtig, um das Therapieansprechen zu dokumentieren, die chirurgische Verfahrenswahl zu planen und (seltene) Fälle von „complete response“ $\mathrm{zu}$ identifizieren. Als bildgebende Verfahren zum Lokalstaging stehen die Endosonographie (EUS) und die Magnetresonanztomographie (MRT) zur Verfügung.

\section{Fragestellung und Methode}

Ziel dieser systematischen Literaturrecherche war die Analyse der diagnostischen Genauigkeit der EUS und der MRT im Restaging beim Rektumkarzinom. Hierzu wurden aus den bekannten medizinischen Datenbanken alle relevanten Publikationen zwischen Januar 1996 und März 2012 extrahiert. Berücksichtigt wurden alle Originalpublikationen mit mindestens 20 eingeschlossenen Patienten, bei denen nach neoadjuvanter RCT ein Restaging mittels MRT und/oder EUS durchgeführt wurde. Zielkriterien waren das T-Stadium (T0 vs. T1-4 bzw. T3/4 vs. T0-2), N-Stadium ( $\mathrm{N}+$ vs. $\mathrm{N}-$ ) sowie der

\section{Ergebnisse}

Insgesamt wurden 24 Studien mit $1201 \mathrm{~Pa}$ tienten in die Metaanalyse eingeschlossen. Mit einer Sensitivität von 82,1 bzw. 87,6\% konnte die MRT und die EUS ein T3/4Tumor (vs. T0-2) mit hoher Wahrscheinlichkeit korrekt vorhersagen, mit leichten Vorteilen für den EUS. Insgesamt war ein Overstaging durch die Bildgebung wahrscheinlicher als ein Understaging. Dagegen konnten sowohl die EUS als auch die MRT ein yT0-Stadium nur in wenigen Fällen korrekt identifizieren (Sensitivität 37,0 bzw. 15,3\%). Ebenso war die diagnostische Genauigkeit der EUS und der MRT im Restaging des Lymphknotenstatus mit einer Sensitivität von 49,8 bzw. $61,6 \%$ eher gering, mit leichten - jedoch nicht signifikanten - Vorteilen für die MRT. Die Sensitivität bzw. Spezifität der MRT in der Beurteilung des CRM war dagegen mit 85,4 bzw. 80,0\% hoch, Ergebnisse hierzu fehlen für die EUS (methodenbedingt).

\section{Diskussion und Fazit des Reviewers}

Die Metaanalyse zeigt in erster Linie, dass ein exaktes Restaging nach neoadjuvanter RCT - u. a. durch therapiebedingte Veränderungen im Gewebe (Fibrose, Ödem, Entzündung, Nekrose) auch für die moderne Bildgebung eine große Herausforderung bleibt. Dies gilt insbesondere für die korrekte Identifikation von komplettem Tumoransprechen (yT0). Hier konnten Habr-Gama und Mitarbeiter jedoch zeigen, dass komplettes Tumoransprechen v. a. eine klinischendoskopische Diagnose ist, und bildgebende Verfahren lediglich der Beurteilung einer residuellen extrarektalen Tumormanifestation dienen [1]. Darüber hinaus weist die Metaanalyse auf die unterschiedlichen diagnostischen Stärken/Schwächen der Bildgebung hin: Die EUS scheint exakter in der Beurteilung der T-Kategorie, wohingegen eine Beurteilung des CRM am besten mit der MRT gelingt. Aus diesem Grund kommen in unserer Klinik bei Karzinomen im unteren und mittleren Rektumdrittel sowohl prätherapeutisch als auch präoperativ immer beide Verfahren zur Anwendung.

\section{Korrespondenzadresse}

\section{Prof. Dr. C.-T. Germer}

Klinik für Allgemein-, Viszeral-, Gefäß- und Kinderchirurgie, Universitätsklinikum Würzburg, Oberdürrbacher Str. 6, 97080 Würzburg germer_c@ukw.de

Interessenkonflikt. J. Reibetanz und C.-T. Germer geben an, dass kein Interessenkonflikt besteht.

\section{Literatur}

1. Habr-Gama A, Perez RO, Wynn G et al (2010) Complete clinical response after neoadjuvant chemoradiation therapy for distal rectal cancer: characterization of clinical and endoscopic findings for standardization. Dis Colon Rectum 53:1692-1698 